\title{
Correlation analysis on serum inflammatory cytokine level and neurogenic pulmonary edema for children with severe hand-foot-mouth disease
}

\author{
Jin-Fang Sun, Hao-Lan Li ${ }^{*}$ and Bao-Xia Sun
}

\begin{abstract}
Objective: This study aims to discuss the correlation between serum inflammatory cytokines and neurogenic pulmonary edema (NPE) in children with severe hand-foot-mouth disease (HFMD).

Methods: A total of 89 patients with severe HFMD were enrolled into this study. These patients were divided into two groups, according to the presence of NPE: central nervous system disease (CNSD) group and NPE group. Serum IL-4, IL-10, IL-6, IL-17, tumor necrosis factor-a (TNF-a), and interferon- $\gamma$ (IFN- $\gamma$ ) levels were measured in patients by enzyme-linked immunosorbent assay at 1, 3, and 5 days after admission. Furthermore, risk factors for NPE were screened using multivariable logistic regression analysis.

Results: IL-6, TNF-a, IL-10, and interferon- $\gamma$ (IFN- $\gamma$ ) levels in the NPE group were higher than in the CNSD group. TNF$a, I L-10$, and IFN- $\gamma$ levels reached a peak on the 3rd day of admission. Age, continuous fever, blood sugar, white blood cell count, and IL-10 were risk factors for the occurrence of NPE in severe HFMD.
\end{abstract}

Conclusion: The dynamic unbalance of inflammatory cytokines is related to the occurrence and progress of NPE.

Keywords: Hand-foot-mouth disease, Cytokines, Dynamic change, Neurogenic pulmonary edema

\section{Background}

Neurogenic pulmonary edema (NPE) is an important complication and a primary cause of death in handfoot-mouth disease (HFMD), and most of these cases die within $12 \mathrm{~h}$ after onset [1]. Research has shown that serum inflammatory cytokine levels significantly increase in severe HFMD patients with NPE [2-4], and is closely correlated to its prognosis [5, 6]. However, there is lack of research on the dynamic change in serum inflammatory cytokines and its correlation to NPE in patients with severe HFMD during the progress of the disease. In the present study, the dynamic changes in inflammatory cytokine levels in patients with severe HFMD after

\footnotetext{
*Correspondence: drlihl16@163.com
}

Department of Infections Disease, Zaozhuang Municipal Hospital,

Leading Road No. 41, Shizhong District, Zaozhuang 277100, Shandong, China admission, who were treated and cured in two hospitals in Henan Province, China from March 2010 to December 2012, were monitored. Furthermore, the correlation between serum inflammatory cytokines and NPE in patients with severe HFMD, and the indicators for predicting NPE, were discussed to provide basis for disclosing the formation mechanism of these severe cases.

\section{Methods \\ General information}

Case selection: pediatric patients with clinically diagnosed severe HFMD, who were admitted at Zhengzhou Children's Hospital and Henan Infectious Hospital from March 2010 to December 2012, were included into the present study. Severe HFMD was clinically diagnosed according to the clinical characteristics, including fever with or without rash, and neurological, respiratory, and circulatory manifestations. All patients were identified 
and treated according to the HFMD Medical Technology Guidance for Medical Institutions (issued by the Ministry of Public Health in 2008) [7]. A signed informed consent was obtained from the parents of children who were clinically diagnosed with severe HFMD and hospitalized, in order to collect specimens from admission. This study was approved by the Medical Ethics Committee of these two hospitals. The treatment for these patients was a combination of intravenous immune globulin (IVIG) and corticoids: IVIG, $q d, 1.0 \mathrm{~g} / \mathrm{kg}$ at a time, continuous use for 2 days; corticoids, bid, $2.0 \mathrm{mg} / \mathrm{kg}$ at a time, the dose was halved after 3 days, and the treatment was stopped after 2 days.

\section{Patients grouping}

According to the occurrence of NPE, the severe HFMD children were divided into two groups: NPE group and central nervous system disease (CNSD) group. The diagnosis criteria for NPE: (1) emergency dyspnea, cyanosis, respiration rate $>30$ breaths per minute, frothy sputum; (2) both lungs have visibly moist rales or howling; (3) chest $\mathrm{X}$-ray revealed increased bronchovascular shadows, a typical butterfly shape, or plaque in the lung; (4) arterial blood gas analysis revealed low $\mathrm{PaO}_{2}$ and high $\mathrm{PaCO}_{2} ;(5)$ no primary heart, lung, or kidney disease; (6) cardiac pulmonary edema and left heart failure was excluded, which was caused by cardiogenic pulmonary edema, and too fast and excessive infusion. CNSD was diagnosed on the basis of the absence of pulmonary edema. Major criteria for neurological involvement: (1) poor mental health, lethargy, headache and vomiting, and body shaking, muscle clonus, nystagmus, ataxia, delayed paralysis, convulsion, etc.; (2) the cerebrospinal fluid examination revealed a clear appearance, increased pressure, increased white blood cell count, mostly mononuclear cells, normal or slightly increased protein level, and normal sugar and chloride levels; (3) MRI revealed that the brainstem and spinal cord gray matter were damaged. The difference in the duration of the disease between the two groups was 1 day.

\section{Data acquisition}

Respiration, pulse, blood pressure, and temperature measurements were first collected within $6 \mathrm{~h}$ of patient admission. Laboratory inspection results were recorded within $72 \mathrm{~h}$ after admission for the first detection. Relevant scales, and clinical classifications and evaluations were respectively conducted by two experienced pediatricians. Furthermore, the disease changes in patients were recorded daily.

\section{Inflammatory cytokine detection}

On the 1st, 3rd, and 5th day after admission of the patient, the serum IL-6, TNF- $\alpha$, IL-4, IL-10, IL-17, and IFN- $\gamma$ levels of each child were determined using an enzyme-linked immunosorbent assay detection kit (Shanghai Joyee Biotechnics Co., Ltd.), according to kit introductions.

\section{Statistical analysis}

For the comparison of continuous variables, a normality test was performed using the Kolmogorov-Smirnov test. Variables that conform to normal distributions were expressed as $\bar{x} \pm s$, otherwise were expressed as medians (such as p25 and p75). Data were verified using calibrated-t, and measurement data that did not conform to normal distributions were analyzed using the Mann-Whitney $U$ test. Count data were presented as a frequency number (\%) and evaluated using $X^{2}$ test. For repeated measurement data, analysis of variance (ANOVA) was used for the comparisons made between or intra-groups. Multivariable analysis was performed using the Logistic regression forward-stepwise method, the variables were disregarded according to the result of the likelihood-ratio test, and risk factors were screened with an significance level of 0.05 for inclusion and significance level of 0.1 for exclusion. All data were analyzed using SPSS 19.0, and the inspection level was set at $\alpha=0.05$ on both sides.

\section{Results}

\section{General information}

In the 89 severe HFMD children, NPE occurred in 42 children (NPE group) and CNSD occurred in 47 children (CNSD group). For the comparison of age, length of hospital stay, time of fever duration, heart rate, positive rate of enterovirus type 71 (EV71), white blood cell count, and blood sugar, there was a significant difference in aspects such as blood sugar $(P<0.05$, Table 1$)$.

\section{The dynamic changes in inflammatory cytokines}

Serum IL-6, TNF- $\alpha$, IL-10, and IFN- $\gamma$ levels were higher in the NPE group than in the CNSD group, and the differences were statistically significant $(P<0.05)$. In addition, serum TNF- $\alpha$, IL-10, and IFN- $\gamma$ levels in the NPE group reached the peak value at the 3rd day of admission (Table 2).

TNF- $\alpha$ level was higher in the NPE group $(52.74 \pm 20.22)$ than in the CNSD group $(33.57 \pm 11.43)$ at the 3rd day of admission, and the difference between these two groups at the 1st (NPE group: $58.19 \pm 20.67$; CNSD group: $54.57 \pm 22.01$ ) and 5th (NPE group: $30.08 \pm 12.20$; CNSD group: $28.92 \pm 10.09)$ day was not statistically significant. Furthermore, the time to peak duration was shorter in the NPE group. In addition, IL-6 (d1: NPE group: $70.56 \pm 17.20$, CNSD group: $65.70 \pm 18.27$; d3: NPE group: $74.80 \pm 20.68$, CNSD 
Table 1 Comparison on demographic and clinical data for NPE and CNSD children patients

\begin{tabular}{|c|c|c|c|}
\hline & NPE group & CNSD group & $P$ value \\
\hline N & 42 & 47 & 0.015 \\
\hline Age (months, $\bar{x} \pm s$ ) & $17.55 \pm 5.12$ & $22.94 \pm 10.37$ & 0.552 \\
\hline Boy (cases, (\%)) & $23(54.76)$ & $30(63.83)$ & 0.257 \\
\hline Body mass $(\mathrm{kg}, \bar{x} \pm s)$ & $11.86 \pm 2.02$ & $12.72 \pm 2.71$ & \\
\hline Residence & & & 0.267 \\
\hline City & $10(23.81)$ & $13(27.66)$ & \\
\hline Rural area & $22(52.38)$ & $22(46.81)$ & \\
\hline Rural-urban fringe zone & $10(23.81)$ & $12(25.53)$ & \\
\hline Length of stay (days) median (P25 and P75) & $8(6,9)$ & $6(5,7)$ & 0.000 \\
\hline Maximum body temperature $\left({ }^{\circ} \mathrm{C}, \bar{x} \pm s\right)$ & $38.89 \pm 0.60$ & $38.73 \pm 0.80$ & 0.480 \\
\hline Duration of fever (days) median (P25 and P75) & $4(4,5)$ & $3(2,4)$ & 0.000 \\
\hline Cardiac rhythm (times/min, $\bar{x} \pm s$ ) & $227.21 \pm 28.23$ & $161.09 \pm 23.31$ & 0.000 \\
\hline EV71-positive cases (\%) & $37(88.10)$ & $29(61.70)$ & 0.024 \\
\hline White blood cell count $(\times 109 / L, \bar{x} \pm s)$ & $13.75 \pm 3.68$ & $12.84 \pm 2.78$ & 0.033 \\
\hline $\operatorname{CRP}(\mathrm{mg} / \mathrm{L}, \bar{x} \pm s)$ & $15.43 \pm 10.31$ & $9.83 \pm 6.28$ & 0.898 \\
\hline Blood sugar (mmol/L, $\bar{x} \pm s)$ & $7.58 \pm 1.83$ & $6.16 \pm 1.14$ & 0.003 \\
\hline IVI therapy cases (\%) & $37(88.10)$ & $38(80.85)$ & 0.786 \\
\hline Hormonal therapy cases (\%) & $40(95.24)$ & $43(91.49)$ & 0.901 \\
\hline
\end{tabular}

CNSD, central nervous system disease; NEP, neurogenic pulmonary edema; CPR, C-reactive protein; EV71, human enterovirus 71; IVIG, intravenous immune globulin

${ }^{a} t$ test; ${ }^{b} X^{2}$ test; ${ }^{c}$ Mann-Whitney $U$ test

Table 2 Dynamic change for serum inflammatory cytokines of NPE and CNSD children patients

\begin{tabular}{|c|c|c|c|c|c|c|c|}
\hline Group & Cases & IL-6 & TNF-a & IL-4 & IL-10 & IL-17 & IFN- $\gamma$ \\
\hline \multicolumn{8}{|l|}{ Group NPE } \\
\hline 1 day after admission & 42 & $70.56 \pm 17.20$ & $58.19 \pm 20.67$ & $20.24 \pm 5.13$ & $64.11 \pm 19.14$ & $55.67 \pm 42.18$ & $56.61 \pm 10.94$ \\
\hline 3 days after admission & 42 & $74.80 \pm 20.68$ & $52.74 \pm 20.22$ & $22.83 \pm 3.63$ & $80.14 \pm 19.20$ & $53.33 \pm 25.22$ & $58.42 \pm 9.17$ \\
\hline 5 days after admission & 42 & $63.73 \pm 23.85$ & $30.08 \pm 12.20$ & $21.28 \pm 4.50$ & $59.92 \pm 20.95$ & $48.10 \pm 27.22$ & $55.31 \pm 8.94$ \\
\hline \multicolumn{8}{|l|}{ Group CNSD } \\
\hline 1 day after admission & 47 & $65.70 \pm 18.27$ & $54.57 \pm 22.01$ & $19.05 \pm 5.78$ & $59.56 \pm 22.85$ & $48.14 \pm 33.26$ & $48.91 \pm 11.73$ \\
\hline 3 days after admission & 47 & $55.99 \pm 13.86$ & $33.57 \pm 11.43$ & $20.38 \pm 4.54$ & $59.68 \pm 16.98$ & $44.81 \pm 28.44$ & $53.11 \pm 8.96$ \\
\hline 5 days after admission & 47 & $49.23 \pm 13.34$ & $28.92 \pm 10.09$ & $19.60 \pm 4.54$ & $42.12 \pm 16.52$ & $34.29 \pm 27.33$ & $45.88 \pm 11.06$ \\
\hline $\begin{array}{l}\text { Value } i \\
F_{\text {interblock }} \text { value ( } P \text { value) }\end{array}$ & & $7.855(0.077)$ & $3.644(0.042)$ & $4.361(0.052)$ & $16.823(0.000)$ & $3.061(0.084)$ & $20.142(0.000)$ \\
\hline$F_{\text {time }}$ value ( $P$ value) & & $1.876(0.177)$ & $13.133(0.001)$ & $0.873(0.354)$ & $14.580(0.001)$ & $1.329(0.254)$ & $3.093(0.045)$ \\
\hline$F_{\text {interaction }}$ value ( $P$ value) & & $2.192(0.145)$ & $0.2919(0.592)$ & $0.152(0.618)$ & $2.612(0.078)$ & $3.736(0.059)$ & $0.513(0.600)$ \\
\hline
\end{tabular}

CNSD, central nervous system disease; NPE, neurogenic pulmonary edema; $F_{\text {interblock }}$ value, It refers to the variation ( $F$ value) between NPE group and CNSD group; $F_{\text {time }}$ value, it refers to the variation of different time $\left(1,3,5\right.$ days) about NPE group or CNSD group; $F_{\text {interaction }}$ value, it refers to the interaction variation of group and time. ANOVA of repeated measurement data was used for the statistical test about the comparisons made between or intra-groups

group: $55.99 \pm 13.86$; d5: NPE group: $63.73 \pm 23.85$, CNSD group: $49.23 \pm 13.34)$ and IFN- $\gamma(\mathrm{d} 1$ : NPE group: $56.61 \pm 10.94$, CNSD group: $48.91 \pm 11.73$; d3: NPE group: $58.42 \pm 9.17$, CNSD group: $53.11 \pm 8.96$; d5: NPE group: $55.31 \pm 8.94$, CNSD group: $45.88 \pm 11.06$ ) were higher in the NPE group than in the CNSD group within 5 days from admission. The IL-10 level reached a peak value at the 3rd day of admission, and the difference between these two groups at the 1st day was not statistically significant. However, this was observed to increase at the 3rd and 5th day. IL-10 mainly has an anti-inflammatory effect in inhibiting the synthesis of inflammatory mediators such as TNF- $\alpha$ and IL- 6 after activation.

\section{Multivariable logistic analysis}

Risk factor evaluation using an unconditional logistic regression equation in a one-time manner and the 
interactions among the factors were used to screen risk factors for NPE occurrence in several severe patients, and the integrated effects of multiple factors on the absolute risk of NPE were estimated (forward-stepwise regression method). Finally, age, continuous fever, white blood cell count, blood sugar, and IL-10 levels were included into the outcome model (Table 3). The simulated fitting degree analysis (Hosmer-Lemeshow detection) revealed that the fitting degree of the model was good $\left(\chi^{2}=5.943\right.$, $P=0.546)$.

\section{Discussion}

NPE is the most severe complication for severe HFMD. Immune research has indicated that HFMD patients, who have a slight symptom, are in a systemic inflammatory response state, and that severe or critical patients are in a compensatory anti-inflammatory response or mixed antagonistic response state [8-10]. Furthermore, inflammatory cytokine levels in HFMD patients with NPE significantly increase, and are closely correlated with the prognosis [2-6]. TNF- $\alpha$ and IL- 6 are proinflammatory mediators [11-13]. TNF- $\alpha$ can cause the "waterfall" inflammatory response of inflammatory mediators, and IL-6 plays a collaboration and amplification role in inflammatory response. The early production of IL-10 is relatively insufficient, which causes TNF- $\alpha$ and IL-6 to be difficult to control at continuously high levels. Furthermore, it also induces inflammatory responses in tissues and organs. However, in the later period, IL-10 continuously increases to enter into the compensatory anti-inflammatory response, which inhibits the immunologic functions of the body. Furthermore, the capability of removing pathogenic microorganisms is decreased. Therefore, IL-10 may have a double-sided effect in severe HFMD disease progress and changes. Furthermore, this is one of the important reasons that cause the progress of the HMFD state, which conforms to the results of domestic and overseas studies [14, 15].

The disease of an NPE child rapidly progresses, and the fatality rate increases when respiratory circulatory

Table 3 Multivariate logistic regression analysis on the risk factors of severe hand-foot-mouth disease children with neurogenic pulmonary edema

\begin{tabular}{lllll}
\hline Variable & SE & OR value & 95\% Cl & P value \\
\hline Constant term & 0.751 & & & 0.000 \\
Age ( $\leq 24$ months) & 0.541 & 3.383 & $1.173-4.799$ & 0.024 \\
Duration of fever ( $>3$ days) & 0.526 & 4.925 & $1.758-3.794$ & 0.002 \\
$\begin{array}{l}\text { White blood cell count } \\
\quad\left(>15.0 \times 10^{9} / \mathrm{L}\right)\end{array}$ & 0.499 & 3.465 & $1.303-5.220$ & 0.013 \\
Blood sugar $(>8.3 \mathrm{mmol} / \mathrm{L})$ & 0.560 & 7.579 & $2.530-12.704$ & 0.000 \\
IL-10 $(>60.00$ eg/L) & 0.110 & 1.228 & $1.007-1.523$ & 0.037
\end{tabular}

$\mathrm{IL}$, interleukin; OR, odds ratio; $95 \% \mathrm{Cl}, 95 \%$ confidence interval failure occurs. Furthermore, even though they survive, the sequela increases. At present, there is a lack of safe and effective vaccines for humans, globally, and there is no antiviral drug with a definite effect. Therefore, it is the key to increase the cure rate and improve the prognosis in severe patients by identifying the main risk factors, and taking corresponding cure measures timely during the early period. In a number of studies, early severe patients have been identified by comparing clinical and laboratory examinations for general and severe HFMD patients [16-18]. In the present study, the risk factors for NPE included the following: age of $\leq 24$ months old, continuous fever, elevated heart rate, Ev71 positivity, elevated peripheral blood leucocyte and blood sugar levels, and the continuous increase in inflammatory cytokines (IL-6, TNF- $\alpha$, IL-10, and IFN- $\gamma$ ). These conform to the results of previous studies [16-18]. The occurrence of NPE in HFMD is closely correlated to brainstem injury [19]. After brainstem injury, it may cause the dysfunction of autonomic nerves and stress response in the body. Autonomic nerve dysfunction may cause vomiting, convulsion, changes in respiratory rhythm, elevated blood sugar, and hyperleukocytosis. When the body is in a stressed state, it may cause the release of multiple active substances from the internal and external brain, sympathetic nerve excitation, release of much catecholamine, and the waterfall-like release of inflammatory mediators. Furthermore, it can also cause permeability to increase in the pulmonary vessel endothelium and NPE [20]. In the present study, multivariable logistic recession analysis was conducted on variables with statistical significance in a single-factor analysis. The analysis revealed that age, continuous fever, peripheral blood leucocyte, blood sugar, and IL-10 are independent risk factors for the occurrence of severe HFMD.

\section{Authors' contributions}

J-FS substantial contributions to the conception and design of the study, the acquisition, analysis, and interpretation of data for the study, and the drafting of the study; $\mathrm{H}-\mathrm{LL}$ and B-XS critical revisions for important intellectual content; J-FS, H-LL, and B-XS final approval of the version to be published; J-FS, H-LL, and B-XS agreement to be accountable for all aspects of the study, ensuring that questions related to the accuracy or integrity of any part of the study are appropriately investigated and resolved. All authors read and approved the final manuscript.

\author{
Acknowledgements \\ None. \\ Competing interests \\ The authors declare that they have no competing interests. \\ Availability of data and materials \\ Not applicable. \\ Consent for publication \\ Obtained form the guardians of all participants.
}


Ethics approval and consent to participate

Obtained from the Ethic Committee of Zaozhuang Municipal Hospital.

\section{Funding}

None.

\section{Publisher's Note}

Springer Nature remains neutral with regard to jurisdictional claims in published maps and institutional affiliations.

\section{Received: 2 July 2017 Accepted: 14 March 2018}

Published online: 03 May 2018

\section{References}

1. Nadel S. Hand, foot, mouth, brainstem, and heart disease resulting from enterovirus 71. Crit Care Med. 2013:41(7):1821-2.

2. Gong X, Zhou J, Zhu W, Liu N, Li J, Li L, Jin Y, Duan Z. Excessive pro-inflammatory cytokine responses of human monocyte-derived macrophages to enterovirus 71 infection. BMC Infect Dis. 2012;12:224.

3. Wang W, Li W, Yang X, Zhang T, Wang Y, Zhong R, Jiao Y, Li T, Jiang T, Tian Y, Wu H. Interleukin-8 is elevated in severe hand, foot, and mouth disease. J Infect Dev Ctries. 2014;8(1):94-100.

4. Wang SM, Lei HY, Liu CC. Cytokine immunopathogenesis of enterovirus 71 brainstem encephalitis. Clin Dev Immunol. 2012;2012:876241.

5. Zhang Y, Liu H, Wang L, Yang F, Hu Y, Ren X, Li G, Yang Y, Sun S, Li Y, Chen $X$, Li $X$, Jin Q. Comparative study of the cytokine/chemokine response in children with differing disease severity in enterovirus 71-induced hand, foot, and mouth disease. PLoS ONE. 2013;8(6):e67430.

6. Zeng M, Zheng X, Wei R, Zhang N, Zhu K, Xu B, Yang CH, Yang CF, Deng C, Pu D, Wang X, Altmeyer R, Leng Q. The cytokine and chemokine profiles in patients with hand, foot and mouth diseases of different severities in Shanghai, China, 2010. PLoS Negl Trop Dis. 2013;7(12):e2599.

7. Ministry of Health of the People's Republic of China. HFMD prevention and control guidance in 2008. Chin J Exp Clin Infect Dis. 2008;2(3):210-3.

8. Li S, Cai C, Feng J, Li X, Wang Y, Yang J, Chen Z. Peripheral T Iymphocyte subset imbalances in children with enterovirus 71-induced hand, foot and mouth disease. Virus Res. 2014;180:84-91.

9. Jiang T, Li SJ, Ouyang WX, Tan YF, Liu FR, Xiao GJ, Tang L, Zhang H. Changes in Thl7 and CD4+CD25+Treg ceHs and their significance among children with hand, foot and mouth disease. Zhongguo Dang Dai Er Ke Za Zhi. 2013;15(12):1113-5.

10. Wei R, Xu L, Zhang N, Zhu K, Yang J, Yang C, Deng C, Zhu Z, De Groot AS, Altmeyer R, Zeng M, Leng Q. Elevated antigen-specific Th2 type response is associated with the poor prognosis of hand, foot and mouth disease. Virus Res. 2013;177(1):62-5.

11. Huang YY, Luo Y, Zhang JS, Wei LJ. Changes of tumor necrosis factor-a, interleukin- $1 \beta$ and cytokine induced neutrophil chemoattractant- 1 in aqueous humor of rabbits with traumatic endophthalmitis. Recent Adv Ophthalmol. 2013;33(8):713-6.

12. Yang YJ, Yang YL, Xu CL. Effects of diabetes mellitus on posterior capsule opacification and its possible mechanism. Recent Adv Ophthalmol. 2014:34(5):474-7.

13. Fu XY, Zou WJ, Yu Q. Effects of doxycycline on IL-1 and TNF-a in rat alkali burn corneas. Recent Adv Ophthalmol. 2014;34(10):915-7.

14. Yang J, Zhao N, Su NL, Sun JL, LV TG, Chen ZB. Association of interleukin 10 and interferon gamma gene polymorphisms with enterovirus 71 encephalitis in patients with hand, foot and mouth disease. Scand J Infect Dis. 2012;44(6):465-9.

15. Han J, Wang Y, Gan X, Song J, Sun P, Dong XP. Serum cytokine profiles of children with human enterovirus 71-associated hand, foot, and mouth disease. J Med Virol. 2014;86(8):1377-85.

16. Li W, Teng G, Tong H, Jiao Y, Zhang T, Chen H, Wu H. Study on risk factors for severe hand, foot and mouth disease in China. PLOS ONE. 2014;9(1):e87603.

17. Yang T, Xu G, Dong H, Ye M, He T. A case-control study of risk factors for severe hand-foot-mouth disease among children in Ningbo, China, 2010-2011. Eur J Pediatr. 2012;171(9):1359-64.

18. Sui JL, Wang XY, Li HT. Case-control study on risk factors of severe handfoot-mouth disease. Pract Prev Med. 2012;19(10):1486-8.

19. Hao B, Gao D, Tang DW, Wang XG, Liu SP, Kong XP, Liu C, Huang JL, Bi QM, Quan L, Luo B. Distribution of human enterovirus 71 in brainstem of infants with brain stem encephalitis and infection mechanism. Fa Yi Xue Za Zhi. 2012;28(2):85-91.

20. Junttila E, Ala-Kokko T, Ohtonen P, Vaarala A, Karttunen A, Vuolteenaho $\mathrm{O}$, Salo T, Sutinen M, Karhu T, Herzig KH, Koskenkari J. Neumgenic pulmonary edema in patients with nontraumatic intracerebral hemorrhage: predictors and association with outcome. Anesth Analg. 2013;116(4):855-61
Ready to submit your research? Choose BMC and benefit from:

- fast, convenient online submission

- thorough peer review by experienced researchers in your field

- rapid publication on acceptance

- support for research data, including large and complex data types

- gold Open Access which fosters wider collaboration and increased citations

- maximum visibility for your research: over $100 \mathrm{M}$ website views per year

At BMC, research is always in progress.

Learn more biomedcentral.com/submissions 